Cinémas

Revue d'études cinématographiques

Journal of Film Studies

Schaeffer, Jean-Marie, Pourquoi la fiction?, Paris, Seuil, 1999, $350 \mathrm{p}$.

\title{
Elitza Dulguerova
}

Volume 12, numéro 1, automne 2001

Le Paysage au cinéma

URI : https://id.erudit.org/iderudit/024873ar

DOI : https://doi.org/10.7202/024873ar

Aller au sommaire du numéro

Éditeur(s)

Cinémas

ISSN

1181-6945 (imprimé)

1705-6500 (numérique)

Découvrir la revue

Citer ce compte rendu

Dulguerova, E. (2001). Compte rendu de [Schaeffer, Jean-Marie, Pourquoi la

fiction?, Paris, Seuil, 1999, 350 p.] Cinémas, 12(1), 169-178.

https://doi.org/10.7202/024873ar d'utilisation que vous pouvez consulter en ligne.

https://apropos.erudit.org/fr/usagers/politique-dutilisation/ 
SCHAEFFER, Jean-Marie, Pourquoi la fiction?, Paris, Seuil, $1999,350 \mathrm{p}$.

N'avons-nous pas tous éprouvé, dans notre quotidien autrement confortable, ce sentiment enivrant mais quelque peu angoissant de perdre soudain les repères de la "réalité »? De ne plus savoir si nous y sommes encore, ou si ce n'est qu'un rêve, qu'un jeu; si notre mensonge n'est pas en train de prendre corps; si ce que nous imitons ne nous transforme pas à son tour; si, excédés par nous-mêmes, nous ne faisons pas notre propre "cinéma"? Bien que de tels états possèdent leur fascination indéniable, ils laissent sur nous la marque du désarroi. Désorientés, nous ressentons un vague sentiment de culpabilité, même sans en connaître la raison. Que se passe-t-il dans ces états liminaires? Quelle place leur attribuer dans notre vie? Si encore c'étaient des manifestations artistiquement assumées ou sublimées, nous aurions au moins eu, d'après Freud, le réconfort d'une reconnaissance de statut social : aux artistes, il leur est permis de... De faire quoi, au juste? De feindre/imiter/mentir/jouer/nous leurrer/se marrer? Qu' est-ce qui leur confere cette prédilection? C'est que la fiction y est à sa place; ailleurs, elle ne serait pas appropriée. Pourquoi la fiction? Parce qu'elle divertit et nous apporte un plaisir esthétique, une satisfaction capable d'enlever le sentiment d'angoisse et d'insécurité. À condition toutefois qu'elle ne quitte pas la sphère artistique et littéraire.

Nous avons ainsi obtenu la moitié de la réponse - ce sentiment d'étrangeté aurait quelque chose à voir avec la fiction. Reste à savoir quoi, comment, pourquoi, quelle est son étendue.

Le livre de Jean-Marie Schaeffer a l'ambition sinon de répondre à toutes ces questions, du moins de les éclaircir. Pourquoi la fiction? est un plaidoyer en défense de la fiction comme une des 
modalités inhérentes à l'être humain, par laquelle il perçoit, apprend, comprend et connaît le monde. C'est aussi, en même temps, une tentative de réviser plusieurs siècles de "fictoclasme ", et de déplacer le débat ancestral de la culture occidentale: Platon ou Aristote? Enfin, c'est aussi une manière de nous réconcilier avec notre propre temps et de démystifier la menace du «tout-virtuel» à l'aide d'arguments à la fois biologiques et anthropologiques, en démontrant l'ubiquité de la fiction dans la vie individuelle et sociale.

\section{Argumentaire}

Le projet de Schaeffer se déploie sur plusieurs volets : renouer avec la part de fiction au quotidien, aussi bien dans les jeux d'enfants, dans les rêveries que dans les jeux numériques; prouver que la fiction est aussi un vecteur de connaissance et désarmer ainsi les définitions réductrices qui en font un épouvantail ; mettre de l'ordre dans une terminologie souvent très chaotique (définir la représentation, différencier le mensonge du leurre, l'imitation de la feintise). Finalement, il s'agit de déculpabiliser l'usage de la fiction, tout en montrant que ce n'est qu'une question d'usage.

Subdivisé en cinq études, le livre commence par démontrer que l'argument antimimétique - dès sa formulation chez $\mathrm{Pla}$ ton et jusqu'aux récentes réticences envers les héroïnes virtuelles comme Lara Croft ou envers l'incidence de la violence télévisuelle sur la criminalité urbaine - repose sur la crainte que l'univers fictionnel peut contaminer l'univers réel. Schaeffer réfute la pertinence de cette opposition entre le réel et le fictionnel et propose de concilier l'approche antimimétique platonicienne avec la reconnaissance de la fiction chez Aristote: retenir de Platon la part de feintise dans la fiction, emprunter à Aristote la conception de la fiction comme modalité de la perception. Le deuxième chapitre, plus technique, clarifie les différents aspects de la mimèsis: l'imitation, la feintise, la représentation et la connaissance, en comparant les activités mimétiques chez les animaux et chez les êtres humains, et conclut que la tendance vers la production fictionnelle mimétique est un des traits distinctifs de l'homme. Schaeffer formule ensuite, dans un troisième 
temps, sa proposition de la fiction comme «feintise ludique partagée " qui procède par "immersion fictionnelle" et amorce une "modélisation du réel ". Le quatrième chapitre étudie les différents usages de la fiction, aussi bien dans le domaine artistique (de la littérature à travers la photographie et la peinture jusqu'au cinéma et aux jeux virtuels) que ludique tout court (jeux d'enfants, rêveries). Le livre se clôt sur une reconnaissance de la fonction esthétique de la fiction, en hommage à Aristote.

Tout au long de cet ouvrage remarquablement rigoureux, des exemples issus du quotidien le plus banal et des données expérimentales tirées des sciences "dures" viennent combler les lacunes de la théorie littéraire et souligner les fondements bioanthropologiques de la fiction. Croisant les études littéraires avec la psychologie cognitive, les études cinématographiques ou encore l'éthologie dans une perspective pragmatique, ce projet expérimental met en avant une théorie générale de la fiction.

\section{Connaissance par la fiction}

La position anti-antimimérique de Schaeffer s'exprime le plus clairement dans son intention de réintégrer la fiction dans le domaine de la connaissance. Son hypothèse est que la valeur cognitive de la fiction se manifeste par voie mimétique. C'est ainsi qu'il retrouve Aristote, pour qui la mimèsis est une des modalités de la connaissance qui produit des mondes nouveaux, c'est-à-dire qui aboutit à une "modélisation cognitive du réel": la fiction n'est pas ici pensée en opposition à une "réalité " quelconque (p. 57-59). Schaeffer appuie son argumentation sur des études en psychologie cognitive, dans le but de démontrer que les représentations que nous nous créons du monde ne sont pas uniquement issues d'un travail cognitif rationnel, mais que la dimension mimétique ludique y joue un rôle très important. Se référant aux recherches sur l'acquisition de la connaissance du réel chez les enfants, Schaeffer (p. 120) explique que:

L'apprentissage mimétique, loin d'être un phénomène secondaire ou marginal, constitue en fait un des quatre types canoniques d'apprentissage, à côté de la transmission culturelle de savoirs explicites, de l'apprentissage individuel par essai et erreur et du calcul rationnel. Et 
dans le domaine des premiers apprentissages de l'enfant, il constitue mème le type le plus répandu. [...] Les enfants s'immergent mimétiquement dans des modèles exemplifiants: ces modèles, une fois assimilés sous forme d'unités d'imitation, de mimèmes, peuvent être réactivés à volonté ultérieurement.

Cet exemple met en évidence un parti pris très important du livre de Schaeffer: la dimension socio-constructive de l'imitation et, par là, de la fiction. Dépassant de loin le domaine restreint de la production littéraire ou artistique, la genèse de l'activité fictionnelle a, selon l'auteur, une fonction sociale. Cette hypothèse n'est bien sûr pas nouvelle: l'on pourrait associer le propos de Schaeffer à une théorie sociologique de la fin du XIX ${ }^{e}$ siècle, également très influencée par le développement de la psychologie expérimentale: De l'imitation, de Gabriel de Tarde (1907). Seulement, un inversement est venu se produire: pour de Tarde, l'imitation comme principe social générateur signait en même temps le verdict d'une société fondée sur la passivité de ses membres, et montrait l'inclination de l'homme à se soumettre à un exemplum magnétisant. Alors que Schaeffer introduit l'élément d'intention dans la production et dans la réception fictionnelle: l'apprentissage mimétique n'est pas aveugle. Au contraire, la conscience de la dimension ludique de la feintise distingue l'être humain de l'animal, et contribue à la fois à son développement individuel (ontogenèse) et générique (phylogenèse). Schaeffer montre que les activités mimétiques peuvent être nécessaires à la survie de l'individu et à son insertion sociale, mais que leur usage peut aussi être plus idiosyncrasique comme dans les cas de l'autoaffection ou de l'autostimulation fictionnelle, et ne pas aboutir à une modélisation cognitive du réel. Si la société (ou la famille) développent des mécanismes de contrôle et de blocage de ces excès de fiction, c'est que le "contrat de la fiction" tel que défini par Schaeffer n'y est pas respecté: il leur manque la con: cience de la dimension ludique et la nécessité du partage social.

\section{Feintise ludique partagée}

Dans sa définition de la fiction, Schaeffer s'appuie sur le concept de John Searle de «feintise partagée », qu'il enrichit par l'in- 
troduction de l'élément ludique (p. 146). Le déterminant "partagée" signale la dimension consensuelle de la production et de la réception des fictions: non seulement suis-je consciente du fait de produire ou de percevoir une fiction (que ce soit en jouant, en rêvant, en lisant un roman, en écrivant une histoire ou en regardant un film), mais tous les autres membres de la société (ou du moins un nombre significatif d'entre eux) sont tout aussi capables de distinguer ce que je produis ou perçois en tant que fiction. C'est dans ce sens que la fiction se distingue du leurre et du mensonge: loin de cacher ses conditions d'énonciation, elle en fait le garant de son existence. Cette transparence consensuelle, qui n'est pas une donnée statique, mais varie en fonction des cultures et des contextes, constitue pour Schaeffer une des conditions de la phylogenèse de la fiction: son ancrage social.

D’un autre côté, la quête délibérée du jeu démarque la fiction de la feintise "sérieuse" (de la tromperie) et résulte selon Schaeffer en une production de représentations auxquelles le spectateur (ou le participant) croit de son libre gré, conscient de la dimension ludique de cette production. Dans ce sens, la fiction differe aussi du mythe où je suis en présence de représentations auxquelles je suis contrainte de croire. Dans le cas des représentations fictionnelles, j'adhère de mon propre gré; en revanche, je ne peux les contrôler qu'en partie, puisque le fonctionnement ludique de la fiction me met en situation d'immersion fictionnelle, par laquelle je retrouve cet état ambigu et inquiétant dont il était question au début du texte.

Il ne faudrait cependant pas conclure qu'il existe des représentations par essence fictionnelles. Pour Schaeffer toutes les représentations ont un statut commun: il ne peut y avoir de différence de nature entre les représentations fictionnelles et celles que l'on appelle factuelles ou référentielles, puisque les deux relèvent d'une même capacité, biologiquement déterminée, de modéliser le monde par des représentations:

[...] la capacité représentationnelle est une structure neurologique qui a été façonnée de telle sorte par la sélection naturelle qu'elle fonctionne comme interface entre notre système nerveux central, d'un côté, l'environ- 
nement extérieur ainsi que nos propres états et actes corporels, de l'autre (p. 153).

Seuls l'usage des représentations et les mécanismes qu'elles activent dans notre système perceptif different et peuvent alors être spécifiques à une modalité fictionnelle de la connaissance.

\section{Immersion fictionnelle}

Schaeffer défend ainsi une conception pragmatique de la fiction: la distinction entre réalité et fiction n'est dès lors pas essentielle mais dépendante des contraintes de l'usage. Lorsque nous sommes en situation d'immersion fictionnelle, nous percevons des représentations comme nous en percevons dans toute autre situation de la vie; toutefois, la fiction déplace notre jugement de distinction entre le vrai, le faux, le possible, et bloque temporairement notre capacité de contrôler avec attention ce que nous percevons (p. 153-155). Elle provoque ainsi un clivage entre notre capacité mentale de percevoir les représentations, et le système de vérification de ces représentations. Pour illustrer ce phénomène de «coupure du courant» lors de l'immersion fictionnelle entre la perception des représentations et leur réorganisation en savoir, Schaeffer se réfere aux analyses de Christian Metz sur la position du spectateur au cinéma. Selon Metz, les fictions cinématographiques mettent le spectateur dans un état de leurre perceptif qui agit à un niveau préattentionnel. Pour Schaeffer, cette immersion fictionnelle des perceptions est typique de toute fiction, bien que rarement prise en compte à cause de la prédominance des modèles littéraires dans les théories de la fiction. L'immersion ne correspond toutefois pas à une identification trompeuse et non critique avec un personnage fictif. En état d'immersion, notre corps est le lieu de "transferts perceptifs" entre la réalité et la fiction; par son activité mimétique, il devient médiateur entre les mondes "vrai " et "fictionnel » (ainsi du spectateur de Titanic qui verse de vraies larmes, p. 193). L'immersion occupe dans la perception fictionnelle la place que les autres situations de perception réservent aux croyances. Schaeffer nuance ainsi l'antinomie entre connaissance et fiction: nous ne savons pas si une chose est "vraie» ou "réelle", mais la croyons telle; il s'agit donc d'une production de vérité, tout 
comme avec la fiction il s'agit d'une modélisation du réel. Porte d'entrée vers la fiction, l'immersion montre qu'il est difficile de maintenir la distinction culturellement établie entre "vérité " et "fiction", qu'elle déplace vers la production de connaissance:

\begin{abstract}
[...] la fiction procède certes à travers des leurres préattentionnels, mais son but n'est pas de nous leurrer, d'élaborer des semblants ou des illusions; les leurres qu'elle élabore sont simplement le vecteur grâce auquel elle peut atteindre sa finalité véritable, qui est de nous engager dans une activité de modélisation (p. 199, c'est moi qui souligne).
\end{abstract}

\title{
Modélisation du réel
}

C'est aussi l'apport du cinéma qui permet à Schaeffer d'orienter sa théorie de la fiction dans une perspective davantage pragmatique et de réfuter les approches sémantiques de la fiction qui la réduisent immanquablement au fictif et au faux-semblant. La position de Schaeffer a le mérite de la clarté: il reproche aux défenseurs d'interprétations sémantiques (de G. Frege à $N$. Goodman) de restreindre la fiction à une sphère régie par ses propres lois, qui ne rejoint à aucun moment la réalité. Schaeffer démontre qu'une telle approche ne saurait inclure le cinéma, mais qu'elle se limite à une conception de la fiction soit comme texte, soit cornme structure (récit). Dans les deux cas, les approches sémantiques ne permettent pas de savoir pourquoi nous avons besoin de fictions, ni pourquoi nous retirons, de leur consommation, un plaisir esthétique. Selon Schaeffer, ces problèmes sont éliminés dès lors que l'on considère la fiction au-delà de l'opposition vrai-fictif, comme un "genre de la réalité" (p. 212) qui modélise un certain type d'appréhension et de connaissance du monde. Ainsi, la fiction apparaît-elle davantage comme un dispositif que l'auteur et le spectateur (ou les participants) sauront reconnaître comme tel, plutôt que comme une propriété du texte/film/jeu.

\section{Médias et dispositifs}

Les dispositifs fictionnels ne recoupent pas nécessairement les différents médias qui véhiculent la fiction (littérature, théâtre, 
cinéma, jeux...). Schaeffer s'oppose ici à une tradition exégétique qui associe depuis Platon et jusqu'à Metz la fiction avec un média particulier (la peinture, la photographie, le cinéma). Puisqu'il n'y a pas de média proprement fictionnel, mais uniquement des usages fictionnels ou factuels des médias, les dispositifs varient en fonction des expériences fictionnelles qui modélisent différents aspects de la réalité (p. 242). Schaeffer distingue sept postures d'immersion fictionnelle dans lesquelles l'imitation porte soit sur des actes du langage, soit sur des événements, soit sur des perceptions (visuelles, auditives, etc.), soit sur des types de comportements. À chaque fois, les différents référents engendrent des modes d'immersion distincts: du jeu proprement mental (dans certaines fictions littéraires) jusqu’à la recherche d'identification comportementale (dans les jeux, par exemple).

Cette classification, qui confronte la théorie schaefferienne de la fiction à des exemples concrets, soulève quelques questions. Un des problèmes qui apparaît clairement dans le cas des représentations visuelles est celui du partage social comme condition d'existence de la fiction: rappelons que celui-ci exige la présence d'une intention claire aussi bien lors de la conception que lors de la perception de la fiction. Ainsi, seules les représentations intentionnellement conçues et perçues comme telles peuvent être considérées comme des fictions. Or, dans le cas des représentations visuelles fixes (peinture, photographie), la fiction est une représentation de deuxième niveau: un paysage allégorique de Poussin est une fiction, selon Schaeffer, parce qu'il introduit une représentation d'une représentation, alors qu'une vue de Delft de Vermeer ne l'est pas, puisqu'elle ne dépasse pas le premier niveau de représentation homologue de la réalité (p. 291). Mais l'on sait que les représentations visuelles, plus que les productions littéraires ou les jeux dont les conventions et mécanismes restent relativement stables au fil des siècles, sont au contraire sujettes à des fluctuations des codes: ce que nous considérons aujourd'hui comme fiction ne l'a pas été nécessairement à son époque, et inversement. L'bistoricité des productions fictionnelles constitue un des points fragiles de la théorie de Schaeffer: si en effet la fiction est une question d'usage qui 
nécessite un partage, resterait-t-elle fiction une fois que le code d'usage est perdu et que la réception est fragmentaire et idiosyncrasique (ainsi des tableaux historiquement et culturellement codés de Poussin ou de toute la tradition de peinture "d'histoire" dont souvent nous ne possédons plus les clés de lecture)? Les modalités de fonctionnement de la fiction telle que définie par Schaeffer sont en revanche facilement perceptibles dans des formes à variabilité historique insignifiante et peu institutionnalisées - tels les jeux d'enfants dont la transmission favorise la constance des mêmes motifs (p. 238).

\section{Comme si...}

Il paraît que la question de la fiction regagne en intérêt aux tournants de siècle. À la fin du XIX siècle, plusieurs théories sociologiques et philosophiques font de la propension humaine à la fiction leur argument principal. Ainsi, G. Agamben (2000) résume-t-il la position du nietzschéen Jules de Gaultier en ces termes: "N'étant rien en lui-même, l'homme peut être seulement s'il agit en faisant comme sil était différent de ce qu'il est (ou plutôt qu'il n'est pas)" (p. 65 ; c'est moi qui souligne). Si une telle position justifiait la nécessité de la fiction par l'insignifiance de l'homme, Jean-Marie Schaeffer en offre un siècle plus tard le versant positif. Constitutive de l'être humain tout au long de son existence (personnelle aussi bien que socio-historique), la fiction selon Schaeffer n'a pas pour objectif de compenser quelque insuffisance, mais se présente comme une relation au monde à part entière. Le besoin du "comme si" ludique est aussi le signe d'un manque fondamental, peu importe si nous l'interprétons avec un signe positif ou négatif. Dans les deux cas, il s'agit d'une différence constitutive de l'être humain, qui gît en lui en attendant d'être activée par voie mimétique. À plusieurs reprises, Schaeffer réfute le cliché qui assimile la fiction à une identification aveugle et soumise à un autre. Dans sa conception, si la tendance à la fiction est inhérente à l'être humain et consacrée par l'usage social, ce n'est pas parce qu'elle lui procure une solution de facilité (telle que l'identification), mais parce qu'elle met au clair une différence. Au lieu de produire une identification, elle est plutôt le signal d'une distanciation, à la 
fois du même (du moi) et de l'autre: une désidentification. Ainsi Schaeffer réfute-t-il les arguments sur les effets maléfiques des arts mimétiques (lors de la mise en scène de la violence, par exemple) en montrant que la fiction ne diminue pas notre conscience de la "réalité », mais agit dans un champ différent, et que "[l]e problème des enfants qui réagissent par un passage à l'acte réside précisément dans le fait qu’ils ont des difficultés à accéder à l'état d'immersion fictionnelle, et donc au "comme si " " (p. 324). Cette affirmation garde toutefois un caractère quelque peu discriminatoire et laisse en effet peu de choix à ceux qui n'ont pas eu suffisamment le goût du jeu dans leur enfance: la propension au "comme si " devient une condition du développement personnel et le lieu d'une "autosélection" au sein de «l'espèce humaine» (p. 327).

Bien qu'il soutienne à plusieurs reprises que la fiction en tant que procédé n'ait pas beaucoup changé depuis la nuit des temps, Schaeffer bâtit sa théorie sur une prise en compte des incidences des nouveaux médias sur notre perception de la fiction. Les exemples issus du cinéma et des jeux numériques lui permettent de "démonter" les théories purement littéraires de la fiction et de rendre compte de ses dimensions psychologique (phénomène de l'immersion fictionnelle), ludique (parenté originaire avec les jeux d'enfants) et cognitive (modélisation du réel). Malgré la lourdeur de son appareil terminologique complexe, l'engagement de Schaeffer pour une réconciliation de la fiction avec les pratiques quotidiennes aussi bien qu'artistiques reste fascinant. Il parvient ainsi à déconstruire deux préjugés toujours présents : l'affirmation du tout-fiction (le simulacre qui a hanté le postmodernisme); et son contraire, l'exclusion "puritaine" de la fiction de la sphère pratique.

Elitza Dulguerova

Université de Montréal

\section{RÉFÉRENCES BIBLIOGRAPHIQUES}

Agamben, 2000 : Giorgio Agamben, Le Temps qui reste, Paris, Rivages, 2000.

de Tarde, 1907: Gabriel de Tarde, Les Lois de limitation. Étude sociologique, Paris, Félix Alcan éditeur, 1907. 